\title{
Data-driven condition monitoring approaches to improving power output of wind turbines
}

\author{
Peng Qian, Xiandong Ma, Dahai Zhang, Junheng Wang
}

\begin{abstract}
This paper presents data-driven approaches to improving active power output of wind turbines based on estimating their health condition. The main procedure includes estimations of fault degree and health condition level, and optimal power dispatch control. The proposed method can adjust active power output of individual turbines according to their health condition and can thus optimize the total energy output of wind farm. In the paper, extreme learning machine (ELM) algorithm and bonferroni interval are applied to estimate fault degree while analytic hierarchy process (AHP) is used to estimate the health condition level. A scheme for power dispatch control is formulated based on the estimated health condition. Models have been identified from supervisory control and data acquisition (SCADA) data acquired from an operational wind farm, which contains temperature data of gearbox bearing and generator winding. The results show that the proposed method can maximize the operation efficiency of the wind farm while significantly reduce the fatigue loading on the faulty wind turbines.
\end{abstract}

Index Terms - Extreme learning machine (ELM), health condition estimation, bonferroni interval, analytic hierarchy process (AHP), condition monitoring, wind turbines.

\section{I.INTRODUCTION}

The wind power industry has grown dramatically in China and worldwide over the past 15 years. In 2015, China has completed installed capacity of $30.5 \mathrm{GW}$, which accounts for $48.4 \%$ of total installation in the world and total installed capacity of wind turbine reached $145 \mathrm{GW}$ by 2015 . With increasing penetration of wind energy, the reliability of power grid effected by wind energy is a challenge [1]. Thus, it is necessary for wind farm to provide stable active and reactive powers to support the frequency and voltage value in the power grid. To fulfill these requirements, the operation of a modern wind farm is required to be much more like a conventional power plant [2]. In this circumstance, keeping each wind turbine to operate in a stable condition is very essential. However, wind turbines are usually installed in harsh conditions, especially for offshore wind farm, which poses a challenge for operation and maintenance [3-6]. Wind turbine is also a complex generation system, as shown in Fig.1; health condition of each component will affect the working efficiency of whole system. The wind turbine usually consists of a number of components and subsystems, including generator, gearbox, bearing, rotor hub and blade, main shaft, mechanical brake, power electronic converter [79]. It is worth noting that health condition of each component or subsystem not only determines the whole turbine operational efficiency, but also affects the power output and thus the power control scheme. This can play a critical role in reliable operation of a large-scale wind power plant when connected to the power grid.

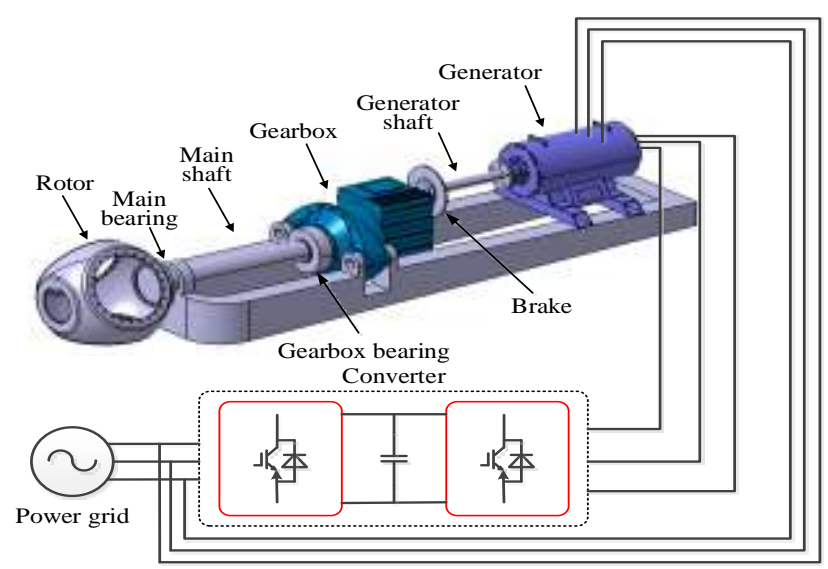

Fig.1 A typical doubly-fed induction generator based wind turbine

Many methods have been proposed to achieve optimizing control of wind farm power output. Minimizing fatigue loading for wind turbine is one of the main objectives [10,11], whereas maximizing energy production of the turbine is another objective [12]. However, little work has considered substantially the influence of health condition of wind turbines on power dispatch control of the wind farm. Generally, each turbine in the farm was assumed to possess a same health condition $[13,14]$. Hence, the power dispatch scheme is usually made based on actual wind speed that indicates power available for the wind turbines to extract; existing active power control algorithms are developed usually based on averaging power assignment or the proportion of actual power output from the turbines in response to the maximum power demand [15].

This paper proposes an optimizing control scheme for active power output of the wind farm based on the estimated health condition. It aims at adjusting power output of the individual turbines according to their health condition to optimize the total energy output of wind farm whilst reducing the fatigue loading on the faulty wind turbine. Consequently, the proposed method can avoid the faulty wind turbines to develop themselves into detrimental ones, which greatly benefit to the operation and maintenance of the wind turbines and hence lower significantly the cost of wind power production.

Three main targets will be achieved in this paper. The first one is associated with fault degree estimation of the turbines while the second one is to estimate health condition level. The third one is related specifically to optimizing control of active power output for the wind farm. A data-driven condition monitoring $(\mathrm{CM})$ method by using extreme learning machine (ELM) algorithm incorporating Bonferroni interval is adopted to achieve fault degree estimation. Then analytic 
hierarchy process (AHP) method is employed to estimate the health condition of turbine's constitutions, which considers multi-criterion factors including fault degree, repair cost and maintenance time. The power dispatch control is then implemented based on the heath condition, aiming at reducing fatigue load of the faulty turbines and maximizing the operation efficiency of those healthy turbines.

Essentially, the aim of the proposed method is to ensure the healthy wind turbines generate power as much as possible while reducing fatigue loads on the sub-healthy turbines. Compared with the previous methods, the proposed methods is able to reduce dramatically the fatigue loads on sub-healthy wind turbines while still satisfying the active power demand from the network operator. One major contribution of the paper is to improve active power dispatch of the wind farm by incorporating the health condition of the individual wind turbines. It is the first time for the paper to integrate all the proposed methods to achieve a control scheme for active power dispatch. Furthermore, AHP is applied for the first time to estimate health condition level taking into account the multi-criterion factors of the key components of the turbines.

The remainder of this paper is organized as follows. The method to estimate fault degree is presented in Section 2. In Section 3, the health condition is estimated based on the AHP. Section 4 describes the optimizing control method for power distribution by means of health condition of the wind turbines The SCADA data and results are discussed in Section 5. Finally, Section 6 contains conclusions and suggestions for further work.

\section{II.FAULT DEGREE ESTIMATION}

Wind turbines usually operate in a hostile environment, especially for the offshore wind farm. When there is a fault in wind turbine, the fault degree is expected to obtain as key information that could help determine control of the power output from the turbines. In this section, the fault degree is estimated by a data driven method by means of extreme learning machine (ELM) in order to obtain the residual signal by comparing actual output data with predicted values. Bonferroni's method is employed in order to improve the confidence intervals.

\section{A. Data-driven Method}

The data-driven method based on ELM, proposed in [16], is used for fault detection. Data gathered from measurements are selected as the inputs for the ELM model to predict the output signals of a physical process. Actual output signals gained from the wind turbine are then compared to the predicted ones for the corresponding input signals. Any differences between them could be caused by changes to the system, and may be caused by the occurrence of a fault.

ELM was proposed by Huang for single hidden layer feed forward neural networks (SLFNs) in 2006 [17]. Compared with the traditional back propagation (BP) neutral network, ELM has advantages of faster training speed and better generalization abilities [18, 19]. In the ELM training process, a finite number of arbitrary distinct samples $N$ that is consisted of $\left(x_{i}, y_{j}\right) \in R^{n} \times R^{m}$ are selected, where $x_{i}$ and $y_{j}$ are an $\mathrm{n} \times 1$ input vector and an $\mathrm{m} \times 1$ output vector, respectively. For this example, the number of neurons $\mathrm{L}$ in the hidden layer is 32; the activation function is represented by $g(x)=\frac{1}{1+e^{-x}}$. It is assumed that the ELM is able to estimate the $N$ training samples with errors that can be too insignificant to be ignored. The algorithm can be represented by the following expression:

$y_{i}=f_{L}\left(x_{i}\right)=\sum_{i=1}^{L} \beta_{i} g\left(\omega_{i}, b_{i}, x_{j}\right) \quad j=1, \ldots, N$.

where $\omega_{i}$ is the weight coefficient vector between the $i$ th hidden node and all $n$ input nodes; $\beta_{i}$ is output weight coefficient vector connecting the $i$ th hidden node and all $m$ output nodes; $b_{i}$ represents the bias of the $i$ th hidden node. $\omega_{i}$ and $b_{i}$ are randomly selected in this paper.

Eq. (1) can be rewritten as below,

$H \beta=Y$

where $H$ is the output matrix of the hidden layer, which can be represented as,

$H=\left[\begin{array}{ccc}g\left(\omega_{1}, b_{1}, x_{1}\right) & \cdots & g\left(\omega_{L}, b_{L}, x_{1}\right) \\ \vdots & \cdots & \vdots \\ g\left(\omega_{1}, b_{1}, x_{N}\right) & \cdots & g\left(\omega_{L}, b_{L}, x_{N}\right)\end{array}\right]_{N \times L}$

$\beta=\left[\begin{array}{c}\beta_{1}^{T} \\ \vdots \\ \beta_{L}^{T}\end{array}\right]_{L \times m}$ and $Y=\left[\begin{array}{c}y_{1}^{T} \\ \vdots \\ y_{N}^{T}\end{array}\right]_{N \times m}$

Hence the $i$ th column of $H$ is the outputs of the $i$ th hidden neuron given input variables $x_{1}, x_{2}, \cdots, x_{n}$. The matrix of output weights, $\beta$ ( $L \times m$ dimensions), can then be calculated by simply finding a matrix $\hat{\beta}$ in order to minimize the error function as given below,

$\min _{\beta}\|H \beta-Y\|$

It is worth noting that the input weights $\omega$ and the hidden layer biases $b$ are not changed during this procedure. The solution is expressed as the following

$\hat{\beta}=H^{+} Y$

Minimizing this function is equivalent to obtaining the unique smallest norm least-squares solution of the linear system in eq. (5). The matrix $H^{+}$is the generalized MoorePenrose inverse of the matrix $H$, which can be found using the singular value decomposition method [20]. The data from a healthy wind turbine is selected as the training dataset to obtain the value of $\hat{\beta}$ for the ELM model. The root mean square error (RMSE) is also used here as a measure of how well the models explain the actual output data. When the value of RMSE is below a predefined threshold ( 0.1 in this 
paper), the accuracy of the ELM model is good enough for testing.

\section{B. Bonferroni interval method}

Although a method relying on residual signals alone can detect faults, it is not able to provide accurate degree evaluation about the failure of components. Thus, it is necessary to quantify accurately fault degree in a condition monitoring system, which is also key information for arranging operation and maintenance.

It is hoped that the information of each component is to be obtained simultaneously when a fault occurs. Therefore Bonferroni interval method, which has more accurate confidence intervals $[21,22]$, is applied to estimate deviation level of each component in this study, as expressed below.

$d_{k}\left(\alpha_{k}\right)=\overline{u_{k}}+t_{N-1}\left(\frac{\alpha_{k}}{2 p}\right) \sqrt{\frac{s_{k k}}{N}}$

where $\overline{\mu_{k}}$ is mean value of the observation $\mu_{k}$. In this paper, the observation $\mu_{k}$ can be the residual signal of gearbox bearing temperature and generator winding temperature, representing the key components to monitor in the wind turbines. $t_{N-1}$ is $t$ distributions widely used in statistics that can be found in the distribution table. $N$ is the sample number for each observation. $S_{k k}$ is diagonal value in the covariance matrix $S$ that is derived from a matrix formed by a set of observations $\mu_{k} ; p$ is the number of the observations used, i.e., the two residual signals of gearbox temperature and generator winding temperature. The parameter $\alpha_{k}$ in equation (6) indicates the confidence level that determines the sample deviation degree $d_{k}\left(\alpha_{k}\right)$; once $\alpha_{k}$ is determined, the value of $t_{N-1}$ can be found from $t$ distribution table [23].

The confidence level $\alpha_{k}$ indicates probability of occurrence of the residual signal within a particular range of values. A smaller $\alpha_{k}$ means higher residual signal values are considered and thus a higher deviation level is identified. If $\alpha_{k}<0.01$ (i.e., the highest $1 \%$ residual values), the monitoring data is considered to indicate a fault in the component [24], while, if $\alpha_{k}$ is larger than a particular value which can be application dependent, the component can be in a debilitating condition. In this case study, $\alpha_{k}=0.25$ (i.e., the highest $25 \%$ residual values) is selected as a threshold value for debilitating condition. It is found that the temperatures during last 3 months over a one-year period of operation increase against active power output. This means about 25\% temperature data deviate from the normal curve, most likely due to aging of the components.

\section{III.HEALTH CONDITION LEVEL ESTIMATION}

The results gained in Section 2 can provide a quantitative value about fault degree of each component in wind turbines. As mentioned above, the wind turbine is a complex system and each component should have different weight of significance in whole system in terms of different maintenance time required and cost caused. Hence, health condition estimation can be regarded as a kind of multicriterion decision-making. In order to optimize power dispatch, it is necessary to adopt a method to solve multicriterion decision-making problem of wind turbines based on their health conditions.

As a well-structured technique for organizing and analyzing complex decisions, analytic hierarchy process (AHP) has been widely used in the field of business, government, shipbuilding, healthcare, industry and education $[25,26]$, because of its reliability and ease of use. Furthermore, the AHP can provide a comprehensive and appropriate model for decision making with different criteria. Thus, it is suitable for applying the AHP method to estimate the health condition of a complex system.

The aim of using AHP method is to determine health condition by accounting for multi-criterion factors. In this paper, three criterion elements are used and defined, namely fault degree, repair cost and maintenance time. Fault degree estimation is described in Section 2, which can provide information of health condition for different components in a wind turbine. Health condition may affect power output performance of the wind turbines and determine whether the turbine should be stopped for maintenance or continue to operate with cautions being taken. Maintenance time and repair cost are also selected as criterion elements, because they play a significant role in determining the availability time, productivity and hence economic benefit of the wind turbines.

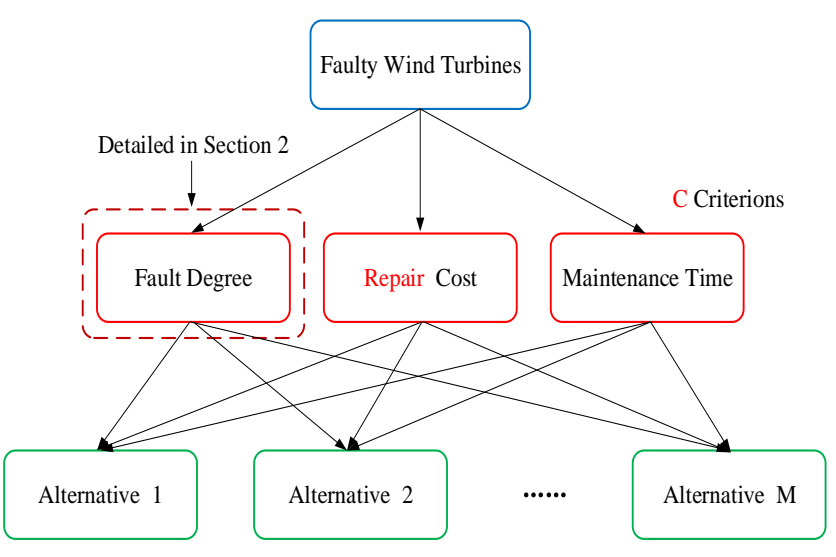

Fig.2 Hierarchical structure for estimating wind turbine health condition

Suppose there are $M$ different alternatives and $C$ different decision criteria. The steps to estimate health condition of the turbines incorporating the AHP are summarized as follows:

1) Define the evaluation criteria and establish a hierarchical structure. Fig. 2 illustrates the hierarchical structure, which contains three major predefined criteria, namely fault degree $C_{1}$, repair cost $C_{2}$ and maintenance time $C_{3}$, and $M$ alternatives. In this paper, $M$ alternatives represent $M$ wind turbines waiting for health estimation.

2) Compose the pairwise criterion elements. Generally, there is an optional number from 1 to 9 , which is usually assigned to a comparison matrix. In this paper, we choose a 
typical three-point performance rating scale, i.e., 1, 5, 9, for significance of criteria. It defines that significance of criteria $C_{1}$ (fault degree), $C_{2}$ (maintenance time), $C_{3}$ (repair cost) are $C_{1}$ high $\left(C_{1}=9\right), C_{2}$ medium $\left(C_{2}=5\right)$ and $C_{3}$ low $\left(C_{3}=1\right)$.

3) Establish the comparison matrix $C_{3 \times 3}$ and the alternative pairwise comparison matrixes $A_{N}(N=1,2$ and 3$)$ Thus, the comparison matrix for three major predefined criteria can be constituted in (7).

$$
\begin{aligned}
& C_{3 \times 3}=\left[\begin{array}{lll}
c_{11} & c_{12} & c_{13} \\
c_{21} & c_{22} & c_{23} \\
c_{31} & c_{32} & c_{33}
\end{array}\right]=\left[\begin{array}{lll}
C_{1} / C_{1} & C_{1} / C_{2} & C_{1} / C_{3} \\
C_{2} / C_{1} & C_{2} / C_{2} & C_{2} / C_{3} \\
C_{3} / C_{1} & C_{3} / C_{2} & C_{3} / C_{3}
\end{array}\right]= \\
& {\left[\begin{array}{ccc}
1 & 9 / 5 & 9 \\
5 / 9 & 1 & 5 \\
1 / 9 & 1 / 5 & 1
\end{array}\right]}
\end{aligned}
$$

The pairwise comparison for $M$ wind turbines is applied with regards to each criterion. A similar method used in equation (7) is therefore adopted to establish alternative pairwise comparison matrixes $A_{N}(N=1,2$ and 3$)$ with respect to the three criteria defined, as described in equation (8).

$A_{N}=\left[a_{i j}\right]_{M \times M} N=1,2,3$

where $a_{i j}$ is pairwise comparison elements between wind turbines regarding each criterion.

The eigenvectors $w_{A 1}, w_{A 2}$, and $w_{A 3}$, corresponding to the maximum eigenvalue of the matrix $A_{N}$, can be calculated by using equations (9-11). Each column element of the comparison matrix $A_{N}$ is normalized as follows

$\overline{a_{\imath \jmath}}=\frac{a_{i j}}{\sum_{i=1}^{M} a_{i j}}$

Then, the line elements of the normalized comparison matrix $A_{N}$ is added together by using equation (10).

$\overline{w_{l}}=\sum_{j=1}^{M} \overline{a_{\imath \jmath}} \quad \mathrm{i}=1,2, \ldots, \mathrm{M}$

The vector $\overline{W_{l}}$ also needs to be normalized via the equation (11)

$w_{i}=\frac{\overline{w_{l}}}{\sum_{j=1}^{M} \overline{w_{J}}} \quad \mathrm{i}=1,2, \ldots, \mathrm{M}$

Consequently, the eigenvectors corresponding to the maximum eigenvalues for matrixes $A_{1}, A_{2}, A_{3}$ are given as below

$$
\begin{aligned}
& w_{A 1}=\left[\begin{array}{llll}
w_{A 11} & w_{A 12} & \cdots & w_{A 1 M}
\end{array}\right] \\
& w_{A 2}=\left[\begin{array}{llll}
w_{A 21} & w_{A 22} & \cdots & w_{A 2 M}
\end{array}\right] \\
& w_{A 3}=\left[\begin{array}{llll}
w_{A 31} & w_{A 32} & \cdots & w_{A 3 M}
\end{array}\right]
\end{aligned}
$$

The vector $w_{c}\left(w_{c}=\left[\begin{array}{lll}w_{c 1} & w_{c 2} & w_{c 3}\end{array}\right]\right)$, an eigenvector corresponding to the maximum eigenvalue of the matrix $C_{3 \times 3}$, can also be calculated by using equations (9-11).
4) Calculate the relative weights for each criterion in order to estimate health condition of the wind turbines via equation (12), which represent the final values of the health condition level.

$\left[\begin{array}{c}w_{1} \\ w_{2} \\ \vdots \\ w_{M}\end{array}\right]=\left[\begin{array}{cccc}w_{A 11} & w_{A 21} & w_{A 31} \\ w_{A 12} & w_{A 22} & w_{A 32} \\ & \vdots & \vdots & \vdots \\ w_{A 1 M} & w_{A 2 M} & w_{A 3 M}\end{array}\right]_{M \times 3} \times\left[\begin{array}{l}w_{c 1} \\ w_{C 2} \\ w_{C 3}\end{array}\right]_{3 \times 1}$

\section{IV.OptIMAL ACTIVE POWER DisPatch}

Wind farm control scheme is designed to make wind farm steady operation by using a separate energy storage system or through de-rated operation of wind turbines [27]. However, utilization of a separate energy storage system would be impossible to apply in a large wind farm, due to high capital investment and high repair cost. Thus, a de-rated operation of wind farm is a practical solution. Generally, power demand from the network operator is less than the maximum available power [10]. Fig. 3 illustrates the schematic diagram of power control scheme for wind farm based on health condition estimation. It contains three parts, namely, network operator (NP), condition monitoring (CM) system and wind farm control (WFC) system. NP monitors the power grid to determine the demand of power output $P^{*}$ to the wind farm; $\mathrm{CM}$ system determines information regarding the health condition $C_{1} C_{2}, \ldots, C_{N}$ of each wind turbine in the farm; WFC allocates the power output to each turbine $\left(P_{1}, P_{2} \ldots P_{N}\right)$ by considering both $P^{*}$ and health condition. For each wind turbine, it may work under its own operation mode such as maximum power point tracing (MPPT) mode, de-rating mode, percentage mode and delta mode [14].

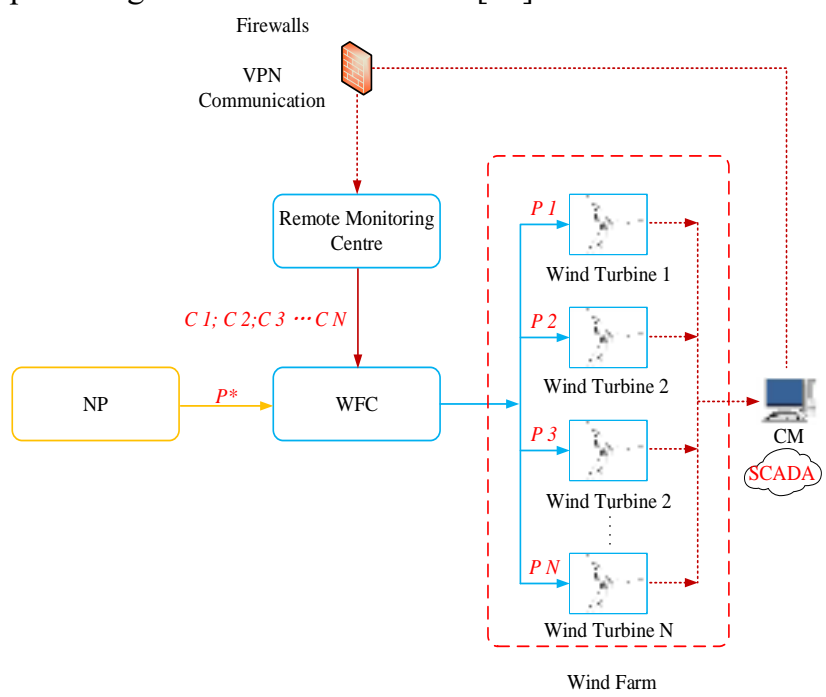

Fig.3 Schematic diagram of power control scheme for wind turbines based on health condition estimation

Generally, a fundamental method has been used to approximate the power curve of the wind turbines [28]. It is approximated by Weibull cumulative distribution function profile that is used in this paper. The wind power curve can be described in (13). 


$$
\begin{aligned}
& P_{\text {wind }}= \\
& \left\{\begin{array}{c}
0 \\
P_{\max }\left(1-e^{-\left(\frac{v}{c}\right)^{k}}\right) \\
P_{\max }
\end{array} \begin{array}{c}
v \leq v_{\text {in }}, v_{\text {out }} \leq v \\
v_{\text {in }}<v<v_{r}
\end{array}\right. \\
& v_{r} \leq v<v_{\text {out }}
\end{aligned}
$$

where $k$ and $c$ are shape and scale parameters, which are used to fit the shape of Weibull distribution profile to the wind power curve.

Fig. 4 compares different power reserve schemes for wind turbines as a function of wind speed. The baseline of power output is obtained via using equation (13). In this figure, there are four turbine operation modes, i.e., baseline mode, derated mode, delta mode and percentage mode. In baseline mode, the wind turbine tries to capture the maximum power from the wind by means of MPPT until it arrives the rated power. If the wind speed is above the rated wind speed but below the cut-out speed, the turbine's output power is capped at the rated power. The de-rate mode is almost the same as baseline mode, but it limits the maximum rated power production to meet wind farm requirements ( $80 \%$ in this case) The percentage mode keeps constant percentage power reserve of the baseline mode ( $80 \%$ in this case) while the delta mode defines wind power production needs to be lower than the available power by a given amount of the baseline mode $(500 \mathrm{KW}$ in this case).

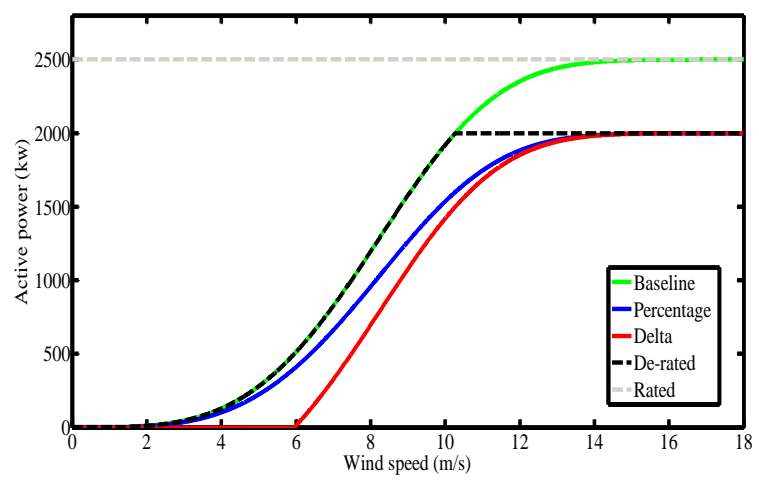

Fig.4 Comparison of different power production schemes for wind turbines as a function of wind speed

From previous research results proposed by Paul A [10], effects of different power generation schemes on wind turbine structural loads can be different. Compared with the baseline mode, other models like de-rated, delta and percentage mode can decrease the fatigue loads on subhealthy wind turbines by limiting maximum power output. Percentage mode has a better performance on reducing the torque loads on drivetrain system than the other modes at a same active power output level. However, percentage mode also suffers higher fatigue loads on blade system than the other modes due to increasing rotating speed of the rotor. The proposed optimal active power control for wind farm based on heath condition of the individual turbines is to reduce mechanical loads acting on the faulty wind turbines while satisfying power output $P^{*}$ demanded by the NP. Thus, the procedures of power dispatch scheme is defined as follows: for the healthy wind turbines, they are set to operate in baseline mode (as shown in green line in Fig 4), which produce maximum power output under variable wind speeds; for the faulty wind turbines, they work in power-reduced mode based on their health conditions. Therefore, the demand of power output $P^{*}$ is defined as below.

$P^{*}=\sum_{i=1}^{N 1} P_{h i}+\sum_{j=1}^{N 2} C_{f j} P_{f j}$

where $N_{1}$ and $N_{2}$ represent the number of the healthy and faulty wind turbines, respectively; $P_{h i}$ is the power output of the $i$ th healthy turbine operating in baseline mode whereas $P_{f j}$ is the power output of the $j$ th faulty turbine working in a power-reduced mode. $C_{f j}$ is the power distribution coefficient of the $j$ th faulty turbine and $C_{f j}=1-w_{f j}, j=1, \ldots, N_{2}$, where $w_{f j}$ is the health condition level of the $j$ th turbine as estimated in Section 3.

When the faulty wind turbines work in a power-reduced mode, the power dispatch control is to make the power output of the faulty wind turbines satisfy the power balance between the network demand and power production of the wind farm. In this paper, power is assigned to the faulty turbines based on their power distribution coefficient considering the health condition level that we discussed in the previous sections. The flow chart of the overall power output scheme is given in Fig.5, accounting for health conditions.

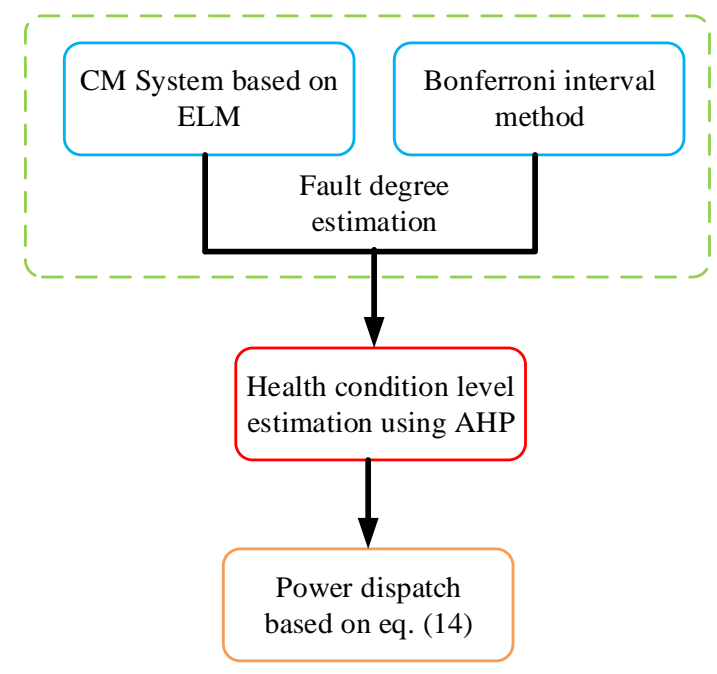

Fig. 5 Flow chart of the proposed method

When the wind turbine is in power-reduced mode, the torque of wind turbine can be calculated as follows.

$\tau= \begin{cases}K \omega^{2} & \omega<\omega_{\text {rated }} \\ \frac{P_{\text {rated }}}{\omega} & \omega=\omega_{\text {rated }}\end{cases}$

where $K$ is a constant depending on the turbine properties; $\omega_{\text {rated }}$ and $P_{\text {rated }}$ are turbine's rated rotation speed and power respectively. 


\section{V.CASE STUDY}

\section{A. SCADA data for fault degree estimation}

The SCADA data used in this paper consist of 128 parameters that contain various temperatures, pressures, vibrations, power outputs, wind speed and digital control signals. In order to reduce the amount of data gathered from the operating wind turbines, SCADA data are usually averaged at 10 minutes' interval, although sampled in the order of seconds. Inactive SCADA data exist due to occasions when a wind turbine is stopped during periods of low and high wind speeds, and due to the occurrence of maintenance periods. Prior to model identification, it is necessary to remove these data when no power is generated. Monitoring techniques based on temperature signals have been developed for fault diagnosis of gearboxes, generators, and power converters. Furthermore, temperature signals can also provide key information on the health condition of mechanical transmission system in wind turbines. The condition monitoring of temperature signal is a proven method to diagnose the faults and predict the residual life of the drivetrain system. Furthermore, the SCADA data acquired from the operational wind farm contain various types of temperature data of the gearbox and generator, which accounts for more than $30 \%$ of the total variables. Thus, the temperature-based condition monitoring method is an effective way to demonstrate the algorithms described in this paper.

In order to validate the proposed fault degree estimation method, gearbox bearing (main-speed shaft bearing connected to the rotor, as shown in Fig.1) and generator winding temperature are selected from SCADA to monitor the condition of gearbox and generator. These signals can be used for feature extraction to show the condition of the gearbox and generator. Therefore, in order to achieve an appropriate model identification, wind speed, ambient temperature and power output are selected as the inputs, while the respective temperature is considered as the output. This multiple-input and single-output (MISO) approach allows a more sensitive detection.

The model predictions for gearbox bearing and generator winding temperatures using the ELM model and Bonferroni interval method are given as follows. Fig. 6 shows the residual signal between the actual temperature and predicted temperature of the gearbox bearing for the faulty turbine during a time duration of 300 hours. The values of $d_{k}(0.01)$ and $d_{k}(0.25)$ for Bonferroni intervals are $9.84{ }^{\circ} \mathrm{C}$ and $6.4{ }^{\circ} \mathrm{C}$ respectively. The residual signal is considered as an anomaly if it is over $d_{k}(0.01)$, while the residual signal is over $d_{k}(0.25)$ but below $d_{k}(0.01)$ is considered as the sub-health condition. It can be seen that the gearbox bearing temperature deviate from the prediction at 120 hours, indicating the onset of a fault. Although the residual signal value between 120 and 280 hours becomes fluctuant but it still lies in the zone of tolerance. However, the residual signal is over $d_{k}(0.01)$ at 295 hours.

Fig. 7 illustrates the residual signal between the actual temperature and predicted temperature of generator winding. The values of $d_{k}(0.01)$ and $d_{k}(0.25)$ for Bonferroni intervals are $41.3{ }^{\circ} \mathrm{C}$ and $25.8{ }^{\circ} \mathrm{C}$ respectively. The temperature of generator winding has some fluctuation around 150 hours; however it is still in the safe area, and at the end of 270 hours the temperature exceeds the value of $d_{k}(0.25)$, indicating a minor fault occurring in the generator.

It is worth mentioning that the data from a healthy wind turbine is selected to train the ELM model and 12.5 days' data (equivalent to 300 hours) when the turbine is in the faulty condition were used to test the model. It is considered that the residual values obtained during training are healthy values and hence are used as a reference for fault detection when tested using the faulty data. The faults occurring in the gearbox and generator winding can be found from an investigation of the SCADA data and from the alarm logs that record fault information, including fault type, fault time and subsequent solutions

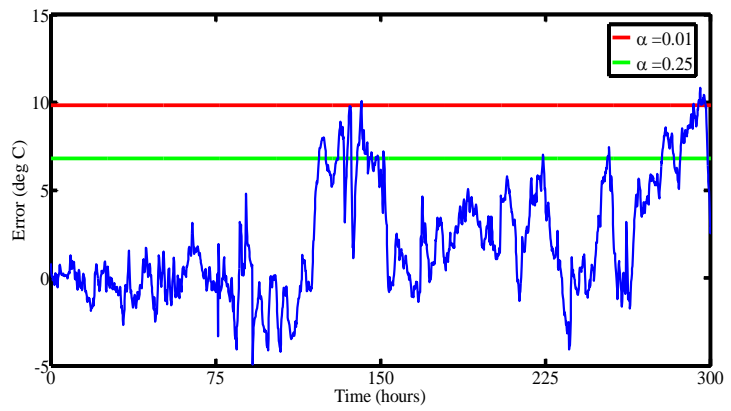

Fig.6 Residual signal of the gearbox bearing temperature between SCADA data and model prediction

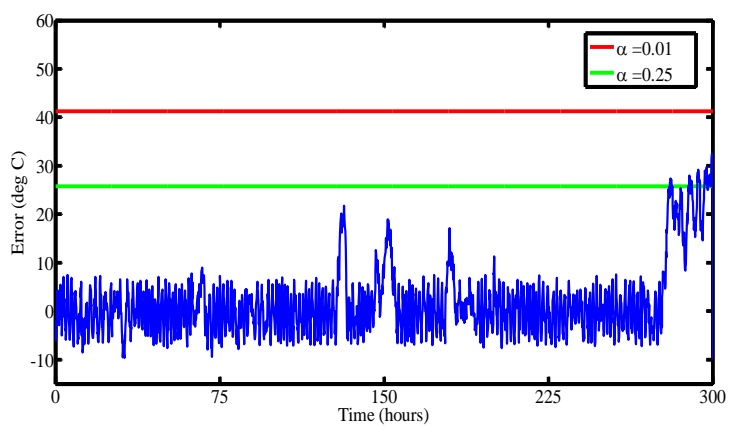

Fig.7 Residual signal of the generator winding temperature between SCADA data and model prediction

After fault degree estimation, AHP is then adopted to estimate health condition considering multiple decision criterions. In this case study, three criterion elements are selected, namely fault degree, repair cost and maintenance time. Table I and Table II provide detailed information about typical repair costs required to repair different subsystems and downtime caused by different subsystems respectively. Here, a lower cost (£5000) is used for the gearbox repair as the gearbox in the faulty turbine experienced only a gear tooth damage.

As mentioned above, it defines that significance of criteria $C_{1}, C_{2}, C_{3}$ are high $\left(\mathrm{C}_{1}=9\right)$, medium $\left(\mathrm{C}_{2}=5\right)$ and low $\left(\mathrm{C}_{3}=1\right)$. We define fault degree has high significance of criterion $\left(C_{1}=9\right)$, maintenance time and repair cost are assumed as medium $\left(\mathrm{C}_{2}=5\right)$ and low $\left(\mathrm{C}_{3}=1\right)$ significance of criterion, respectively. From reference [29], it illustrates that the lost production, while primarily depending on the maintenance 
time, contributes more than the repair cost in terms of the O\&M costs. Therefore, maintenance time is a more important factor to consider than the repair cost. Both maintenance time and repair cost are determined by fault degree of the component, thus fault degree has the highest significance of criterion.

Meanwhile, we also define that the residual signal over $d_{k}(0.01)$ from ELM model is high $\left(\mathrm{A}_{13}=9\right)$; the value between $d_{k}(0.01)$ and $d_{k}(0.25)$ is medium $\left(\mathrm{A}_{12}=5\right)$; and below $d_{k}(0.25)$ is low $\left(\mathrm{A}_{11}=1\right)$. The downtime of subsystems over 10 days is high $\left(\mathrm{A}_{23}=9\right)$; the downtime of subsystems between 10 days and 5 days is medium $\left(\mathrm{A}_{22}=5\right)$, and below 5 days is low $\left(\mathrm{A}_{21}=1\right)$. The repair cost of subsystems over $£ 30000$ is high $\left(A_{33}=9\right)$; the repair cost of subsystems between $£ 30000$ and $£ 10000$ is medium $\left(A_{32}=5\right)$, and below $£ 10000$ is low $\left(A_{31}=1\right)$. Six $2.1 \mathrm{MW}$ wind turbines are selected including four healthy wind turbines and two faulty wind turbines. The fault characteristics of the two faulty wind turbines (one with bearing fault while another with winding fault) are given in Fig.6 and Fig.7. Thus, through the proposed AHP method, it can be obtained that the weight of the wind turbine with a gearbox fault is 0.6924 via using equations (9-13); meanwhile, the weight of the wind turbine with a generator fault is 0.3076 . This means that the wind turbine with a gearbox fault is more significant than the wind turbine with a winding fault.

TABLE I

TYPICAL REPAIR COST FOR DIFFERENT REPAIR TYPES [29]

\begin{tabular}{llr}
\hline \hline Components & Repair type & Cost $(£)$ \\
Gearbox & Repair & 5000 \\
Generator & Repair & 50000 \\
Converter & Repair & 12000 \\
Pitch actuator & Repair & 8000 \\
Bearing & Repair & 5000 \\
Blade & Repair & 4000 \\
\hline \hline
\end{tabular}

TABLE II

DOWNTIME CAUSED BY DIFFERENT SUBSYSTEMS [7]

\begin{tabular}{cc}
\hline \hline Components & Downtime (days) \\
\hline Gearbox & 14 \\
Generator & 3 \\
Converter & 6 \\
Pitch actuator & 4 \\
Bearing & 11 \\
Blade & 4 \\
\hline \hline
\end{tabular}

\section{B. Optimizing active power dispatch scheme}

The proposed method is now compared to the current practice, i.e., the power dispatch for healthy and sub-healthy wind turbines are treated equally. Fig. 8 illustrates the wind speed for a turbine in the wind farm within one day as an example. According to the wind speed, the power output based on different power control schemes as shown in Fig. 4 are then calculated. In this paper, we assume that the network operator adopts $80 \%$ percentage mode and thus we just compare the percentage power control and the baseline power control scheme under the given wind conditions. The results are shown in Fig. 9, where the red line and blue line describe the power output of wind turbines operating in the baseline mode and $80 \%$ power reserve mode, respectively.

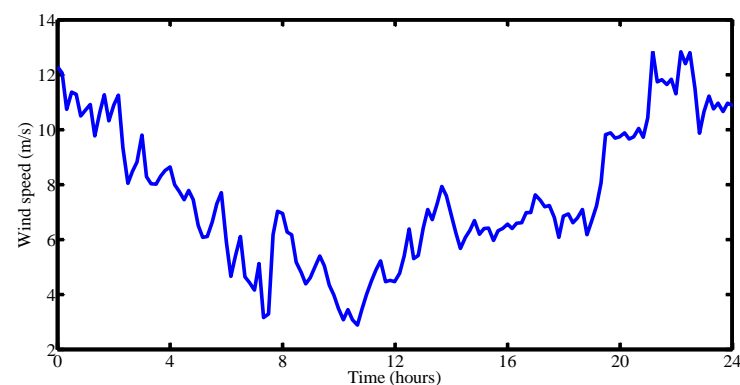

Fig. 8 Wind speed for a wind turbine within one day

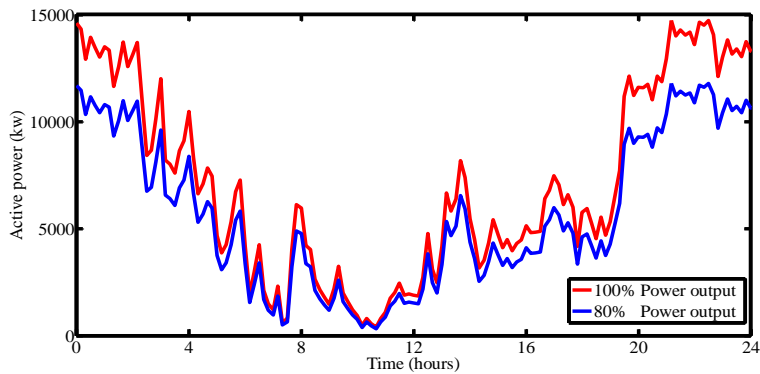

Fig.9 Comparison of the percentage power control (80\%) and the baseline power control under the given wind condition

Fig. 10 provides power output of the six wind turbines (wind turbine 5 with a gearbox fault and wind turbine 6 with a generator fault) following $80 \%$ percentage mode power control. It is worth noting that the power output for the wind turbines has some minute differences under the given wind speed condition, due to the wake effect in wind farm that have been included in the data due to different geometrical locations where wind turbines are installed [30]. The torque of generator for each wind turbine can be calculated by using equation (15) from [7] and is shown in Fig. 11, in response to power demand to the farm. It can be seen that the generator torque for each turbine is almost same; thus this control scheme does not benefit to reduce mechanical load acting on these faulty turbines.

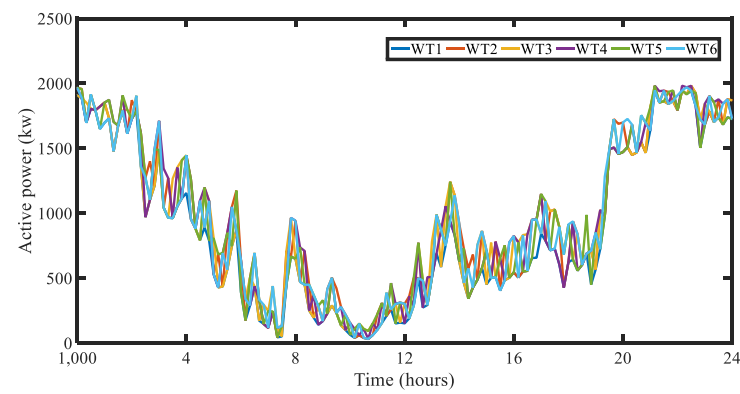

Fig.10 Power output from the turbines using the conventional proportional allocation method 
Fig. 12 illustrates the proposed power output control of the wind farm based on the estimated health condition. In this case, four healthy turbines operate in the baseline mode, and two faulty turbines are in the reducing mode. The power generation for faulty turbines adopts power-reduced mode (wind turbine 5 allocates less power due to a more detrimental gearbox fault) following principle of the equation (14). The wind turbine with a gearbox fault reduces $56 \%$ power generation while the wind turbine with a generator fault decreases $24 \%$ power generation. It can be seen that four healthy turbines operating in the baseline mode produce power at their maximum possible capacities in order to meet demand specified by the network operator. Meanwhile, the two faulty turbines work in the power-reduced mode in order to reduce mechanical load while still balancing network demand and power output from the farm. Fig. 13 describes corresponding torque of the generators based on the proposed method. Clearly, it can be seen that generator torque of the faulty turbines significantly decrease, due to the reduced power output.

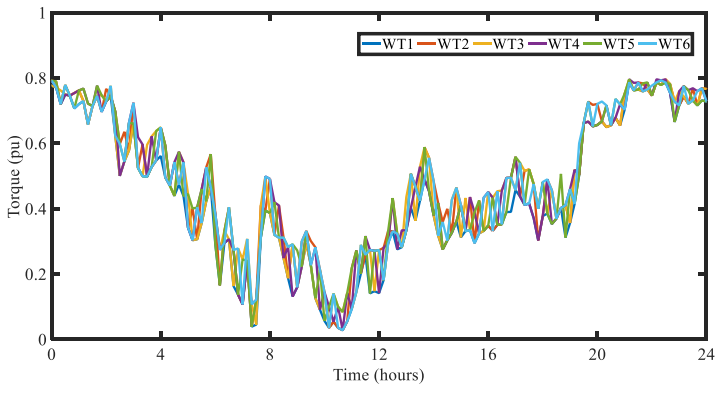

Fig.11 Corresponding torque outputs from the turbines using the conventional proportional allocation method

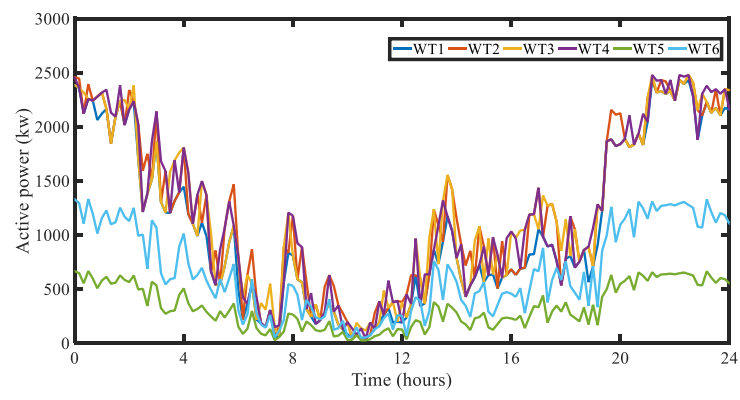

Fig.12 Active power outputs from turbines using the proposed optimizing power output control

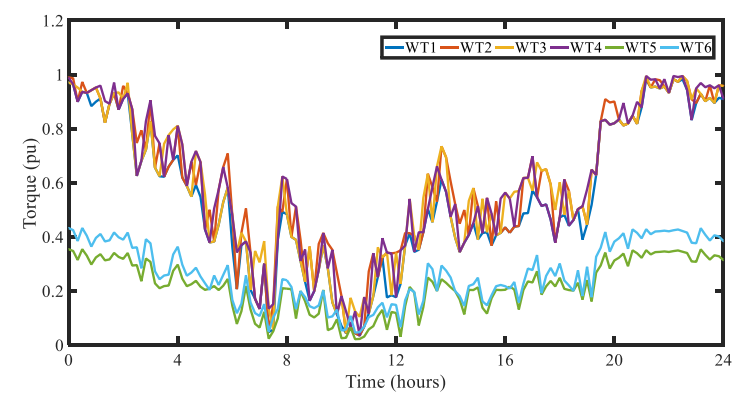

Fig. 13 Corresponding torque outputs from turbines using the proposed optimizing power output control

\section{VI.CONCLUSION}

This paper proposes a control scheme for power output of the wind farm based on the health condition of wind turbines. In order to demonstrate effectiveness of the proposed method, two faulty turbines and four healthy turbines are selected in the case study. Compared with the conventional wind farm control scheme, the proposed method estimates health condition of the individual wind turbines by using data-driven condition monitoring approaches and AHP to determine the health condition levels. Power dispatch control is then made to ensure the healthy wind turbines generate power at their maximum possible capacities and, in the meantime, reduce fatigue loads on the sub-healthy and faulty wind turbines. Through the case study, it shows that the proposed method can significantly reduce torque acting on the faulty wind turbines whilst still satisfying total power output demanded to the wind farm. More specifically, the torque acting on the gearbox can be reduced by average to $55 \%$ of the traditional method for the faulty turbines. The value of $55 \%$ is obtained by comparing the average gearbox torque of the traditional method with the average gearbox torque of the proposed method. This would considerably be beneficial to prolong life-span and economic benefit of the wind turbines. Future work includes identification of health condition of the wind turbines in a timely manner and report back to the controller with appropriate control objectives to achieve an online realtime power dispatch control of the wind farm.

\section{REFERENCE}

[1] W. Yang, P. J. Tavner, and W. Tian, "Wind Turbine Condition Monitoring Based on an Improved Spline-Kernelled Chirplet Transform," IEEE Transactions on Industrial Electronics, vol. 62, pp. 6565-6574, 2015.

[2] M. Tsili and S. Papathanassiou, "A review of grid code technical requirements for wind farms," IET Renewable Power Generation, vol. 3, p. 308, 2009.

[3] P. Cross and X. Ma, "Nonlinear system identification for model-based condition monitoring of wind turbines," Renewable Energy, vol. 71, pp. 166-175, 2014.

[4] W. Zhang and X. Ma, "Simultaneous Fault Detection and Sensor Selection for Condition Monitoring of Wind Turbines," Energies, vol. 9, p. 280, 2016.

[5] J. Wang, F. Cheng, W. Qiao, and L. Qu, "Multiscale Filtering Reconstruction for Wind Turbine Gearbox Fault Diagnosis Under Varying-Speed and Noisy Conditions," IEEE Transactions on Industrial Electronics, vol. 65, pp. 4268-4278, 2018.

[6] B. Lu, Y. Li, X. Wu, and Z. Yang, "A Review of Recent Advances in Wind Turbine Condition Monitoring and Fault Diagnosis," in 2009 IEEE Power Electronics and Machines in Wind Applications, 2009.

[7] W. Qiao and D. Lu, "A Survey on Wind Turbine Condition Monitoring and Fault Diagnosis;Part I: Components and Subsystems," IEEE Transactions on Industrial Electronics, vol. 62, pp. 6536-6545, 2015.

[8] F. Cheng, Y. Peng, L. Qu, and W. Qiao, "Current-based fault detection and identification for wind turbine drivetrain gearboxes," IEEE Transactions on Industry Applications, vol. 53, pp. 878 - 887, 2017. 
[9] F. Cheng, L. Qu, and W. Qiao, "Fault Prognosis and Remaining Useful Life Prediction of Wind Turbine Gearboxes Using Current Signal Analysis," IEEE Transactions on Sustainable Energy, vol. 9, pp. 157-167, 2018.

[10] H. Zhao, Q. Wu, Q. Guo, H. Sun, and Y. Xue, "Distributed Model Predictive Control of a Wind Farm for Optimal Active Power ControlPart I: Clustering-Based Wind Turbine Model Linearization," IEEE Transactions on Sustainable Energy, vol. 6, pp 831-839, 2015.

[11] Y. Jeong, K. Johnson, and P. Fleming, "Comparison and testing of power reserve control strategies for grid-connected wind turbines," Wind Energy, vol. 17, pp. 343-358, 2014.

[12] y. li, Z. Xu, and K. Meng, "Optimal Power Sharing Control of Wind Turbines," IEEE Transactions on Power Systems, pp. 1-1, 2016.

[13] S.-h. Hur and W. E. Leithead, "Adjustment of wind farm power output through flexible turbine operation using wind farm control," Wind Energy, vol. 19, pp. 1667-1686, September 2016.

[14] P. A. Fleming, J. Aho, A. Buckspan, E. Ela, Y. Zhang, V. Gevorgian, et al., "Effects of power reserve control on wind turbine structural loading," Wind Energy, vol. 19, pp. 453-469, 2016.

[15] P. Sørensen, A. D, F. Iov, F. Blaabjerg, and M. H. Donovan, "Wind farm models and control strategies," Risø National LaboratoryAugust 2005.

[16] P. Qian, X. ma, and P. Cross, "Integrated data-driven modelbased approach to condition monitoring of the wind turbine gearbox," IET Renewable Power Generation, 2017.

[17] H. Guang-Bin, Z. Qin-Yu, K. Z. Mao, S. Chee-Kheong, P. Saratchandran, and N. Sundararajan, "Can threshold networks be trained directly?," IEEE Transactions on Circuits and Systems II: Express Briefs, vol. 53, pp. 187-191, 2006.

[18] N. Y. Liang, G. B. Huang, P. Saratchandran, and N. Sundararajan, "A fast and accurate online sequential learning algorithm for feedforward networks," IEEE Trans Neural Netw, vol. 17, pp. 1411-23, Nov 2006.

[19] R. Zhang, Y. Lan, G. B. Huang, Z. B. Xu, and Y. C. Soh, "Dynamic extreme learning machine and its approximation capability," IEEE Trans Cybern, vol. 43, pp. 2054-65, Dec 2013.
[20] A. Grigorievskiy, Y. Miche, M. Käpylä, and A. Lendasse, "Singular Value Decomposition update and its application to (Inc)OP-ELM," Neurocomputing, vol. 174, pp. 99-108, 2016.

[21] M. Ghasemi Hamed, R. Alligier, and D. Gianazza, "High Confidence Intervals Applied to Aircraft Altitude Prediction," IEEE Transactions on Intelligent Transportation Systems, pp. 1-13, 2016 [22] T.-R. Tsai, W.-Y. Sung, Y. L. Lio, S. I. Chang, and J.-C. Lu, "Optimal Two-Variable Accelerated Degradation Test Plan for Gamma Degradation Processes," IEEE Transactions on Reliability, vol. 65 , pp. $459-468,2016$.

[23] K. Krishnamoorthy and T. Mathew, Statistical Tolerance Regions: Theory, Applications, and Computation. NJ, USA: Wiley, 2009.

[24] P. Bangalore and L. B. Tjernberg, "An Artificial Neural Network Approach for Early Fault Detection of Gearbox Bearings," IEEE Transactions on Smart Grid, vol. 6, pp. 980-987, 2015.

[25] N. Liu, J. Zhang, H. Zhang, and W. Liu, "Security Assessment for Communication Networks of Power Control Systems Using Attack Graph and MCDM," IEEE Transactions on Power Delivery, vol. 25 , pp. 1492-1500, 2010.

[26] H. Tanaka, S. Tsukao, D. Yamashita, T. Niimura, and R. Yokoyama, "Multiple Criteria Assessment of Substation Conditions by Pair-Wise Comparison of Analytic Hierarchy Process," IEEE Transactions on Power Delivery, vol. 25, pp. 3017-3023, 2010.

[27] Z. Lubosny and J. W. Bialek, "Supervisory Control of a Wind Farm," IEEE Transactions on Power Systems, vol. 22, pp. 985-994, 2007.

[28] H. Long, L. Wang, Z. Zhang, Z. Song, and J. Xu, "Data-Driven Wind Turbine Power Generation Performance Monitoring," IEEE Transactions on Industrial Electronics, vol. 62, pp. 6627-6635, 2015. [29] B. Le and J. Andrews, "Modelling wind turbine degradation and maintenance," Wind Energy, vol. 19, pp. 571-591, 2016.

[30] M. Martini, R. Guanche, J. A. Armesto, I. J. Losada, and C. Vidal, "Met-ocean conditions influence on floating offshore wind farms power production," Wind Energy, vol. 19, pp. 399-420, 2016. 